\title{
Algorithmic Meta-Theorems
}

\author{
Stephan Kreutzer \\ Oxford University Computing Laboratory, \\ stephan.kreutzer@comlab.ox.ac.uk
}

Algorithmic meta-theorems are algorithmic results that apply to a whole range of problems, instead of addressing just one specific problem. This kind of theorems are often stated relative to a certain class of graphs, so the general form of a meta theorem reads "every problem in a certain class $\mathcal{C}$ of problems can be solved efficiently on every graph satisfying a certain property $\mathcal{P}$ ". A particularly well known example of a meta-theorem is Courcelle's theorem that every decision problem definable in monadic second-order logic (MSO) can be decided in linear time on any class of graphs of bounded tree-width [1].

The class $\mathcal{C}$ of problems can be defined in a number of different ways. One option is to state combinatorial or algorithmic criteria of problems in $\mathcal{C}$. For instance, Demaine, Hajiaghayi and Kawarabayashi [5] showed that every minimisation problem that can be solved efficiently on graph classes of bounded tree-width and for which approximate solutions can be computed efficiently from solutions of certain sub-instances, have a PTAS on any class of graphs excluding a fixed minor. While this gives a strong unifying explanation for PTAS of many problems on H-minor free graphs, the class of problems it defines is not very natural. In particular, it may require some work to decide if a given problem belongs to this class or not.

Another approach to define meta-theorems is therefore based on definability in logical systems, e.g. to consider the class of problems that can be defined in first-order logic. For instance, related to the above example, a result by Dawar, Grohe, Kreutzer and Schweikardt [4] states that every minimisation problem definable in first-order logic has an EPTAS on every class of graphs excluding a minor. While the actual complexity bounds obtained in this way may not live up to bounds derivable for each individual problem, the class of problems described in this way is extremely natural and for many problems their mathematical formulation already shows that they are first-order definable. Consider, e.g., the definition of a dominating set.

Such meta-theorems based on definability in a given logic have received much attention in the literature. For instance, for the case of decision problems, it is has been shown that every problem definable in monadic second-order logic can be decided in polynomial time on graph classes of bounded clique width (see e.g. [2]). For first-order logic (FO), Seese [14] showed that every FO-definable decision problem is solvable in linear time on graph classes of bounded degree. This has then been extended to planar graphs and, more generally, graph classes of bounded local tree-width by Frick and Grohe [7] and to H-minor free graphs by Flum and Grohe [6]. Finally, Dawar, Grohe, Kreutzer generalised these results even further to classes of graphs locally excluding a minor [3]. 
For optimisation problems, well-known meta-theorems employing logic can been found in the work on MAXSNP by Papadimitriou, Yannakakis and also Kolaitis, Thakus (see e.g. $[13,10,11]$ ) or in other syntactical approaches to metatheorems as in, e.g., [9].

While meta-theorems based on logical definability give strong algorithmic results for natural classes of problems, this often comes at the price of sacrificing efficiency in the algorithms derived from them. For instance, while the linear time bound $\mathcal{O}(n)$ of Courcelle's theorem on MSO-properties of graph classes of bounded tree-width is optimal, the constants hidden in the $\mathcal{O}$-notation are horrific and prevent any practical applications of the theorem. It is clear, thus, that the benefit from algorithmic meta-theorems does not lie within immediate applications or (practically) efficient algorithms. Instead they help understand the algorithmic theory of certain graph classes and form an efficient tool for establishing that a problem is tractable on a certain class of graphs or structures.

In this talk I will survey algorithmic meta-theorems with emphasis on motivation and future directions. While the focus will be on decision problems, I will also discuss logical approaches to optimisation problems. Recent surveys on meta-theorems can be found in [8] and also [12].

\section{References}

1. B. Courcelle. Graph rewriting: An algebraic and logic approach. In J. van Leeuwen, editor, Handbook of Theoretical Computer Science, pages 194 - 242. Elsevier, 1990.

2. B. Courcelle, J. Makowsky, and U. Rotics. Linear time solvable optimization problems on graphs of bounded clique-width. Theory Comput. Systems, 33:125-150, 2000.

3. A. Dawar, M. Grohe, and S. Kreutzer. Locally excluding a minor. In Proc. of the 22nd IEEE Symp. on Logic in Computer Science, pages 270 - 279, 2007.

4. A. Dawar, M. Grohe, S. Kreutzer, and S. Schweikardt. Approximation schemes for first-order definable optimisation problems. In Proc. of the 21st IEEE Symp. on Logic in Computer Science, pages 411 - 420, 2006.

5. E. Demaine, M. Hajiaghayi, and K. Kawarabayashi. Algorithmic graph minor theory: Decomposition, approximation, and coloring. In Symposium on Foundations of Computer Science (FOCS), pages 637-646, 2005.

6. J. Flum and M. Grohe. Fixed-parameter tractability, definability, and model checking. SIAM Journal on Computing, 31:113 - 145, 2001.

7. M. Frick and M. Grohe. Deciding first-order properties of locally tree-decomposable structures. Journal of the ACM, 48:1184 - 1206, 2001.

8. M. Grohe. Logic, graphs, and algorithms. In T.Wilke J.Flum, E.Grädel, editor, Logic and Automata History and Perspectives. Amsterdam University Press, 2007.

9. S. Khanna and R. Motwani. Towards a syntactic characterization of PTAS. In Proc. of STOC'96, pages 329-337, 1996.

10. Ph.G. Kolaitis and M.N. Thakur. Logical definability of NP optimization problems. Information and Computation, 115(2):321-353, 1994.

11. Ph.G. Kolaitis and M.N. Thakur. Approximation properties of NP minimization classes. Journal of Computer and System Sciences, 50:391-411, 1995.

12. S. Kreutzer. Finite model-theory of tree-like structures. submitted, available from http://web.comlab.ox.ac.uk/oucl/work/stephan.kreutzer/publications.html. 
13. C.H. Papadimitriou and M. Yannakakis. Optimization, approximation, and complexity classes. Journal of Computer and System Sciences, 43:425-440, 1991.

14. D. Seese. Linear time computable problems and first-order descriptions. Mathematical Structures in Computer Science, 2:505 - 526, 1996. 